\title{
The advisability of using combustion chambers with a toroidal recirculation-mixing zone in small-sized gas turbine engines
}

\author{
Michael Yurievich Orlov ${ }^{1}$, Oleg Vladimirovich Kolomzarov ${ }^{1}$, Vladislav Mikhailovich \\ Anisimov $^{11,}$, Nikita Igorevich Gurakov ${ }^{1}$ and Nikolai Sergeevich Mironov ${ }^{1}$ \\ ${ }^{1}$ Samara National Research University, Heat engine Department, Samara, 443086, Russia
}

\begin{abstract}
Abstarct. The interrelations between the size of the gas turbine engine (GTE) and the size and workflow of the combustion chamber (CC) were considered. On the base of analysis of workflow organization the design of $\mathrm{CC}$ with toroidal recirculation mixing zone was proposed. The theoretical justification of chosen design was carried out on the base of comparison of combustion volumes, which formed in traditional $\mathrm{CC}$ with swirlers and in proposed CC. The comparison of combustion volumes, schematic display of combustion zones with a discrete flame and a combined combustion zone were given.
\end{abstract}

A large number of different requirements can be offered to small-sized GTE (weight, size, durability, level of environmental characteristics, etc.) due to the wide range of tasks it solve. The fulfillment of most of these requirements is related to the choice of the combustion chamber design and is one of the most important problems of preliminary design.

The feature of small-sized engines is its more strict interrelationships between workflow parameters and design. As a result, in such cases, a slight change in geometry can cause a flow reversal. Therefore, with a reduction of the size of the engine relative to the prototype, its combustion chamber cannot be reduced by simply scaling its elements, because of the changes in the preparation of fuel-air mixture, the organization of the cooling system and air mixing.

When considering the combustion chambers of small-sized engines, it is important to determine the meaning of the term "small size", because of different approaches.

The analysis of these criterions shows that, in common case, the engine with air flow rate within the range $1 \leq G_{\mathrm{f}} \leq 10 \mathrm{~kg} / \mathrm{s}$ can be referred to the small-sized engines. For impeller machines of small-sized GTE is characterized by the decrease of power efficiency, and its combustion chamber is characterized by a significant reduction in geometric size and workflow parameters (inlet pressure, outlet temperature, combustion efficiency). The characteristic feature of these engines is the presence of the last centrifugal or axialcentrifugal compressor stage and, as a consequence, the availability of various combustion chamber designs.

As parameters characterizing the dimension, the following applies:

\footnotetext{
${ }^{1}$ Corresponding author: vradik@mail.ru
} 
- $\quad$ air flow rate reduced by parameters beyond the compressor [1]:

$$
G_{\mathrm{f} \text { red }}=G_{\mathrm{f}} \frac{101325}{p_{\mathrm{c}}^{*}} \sqrt{\frac{T_{\mathrm{c}}^{*}}{288}} ;
$$

- $\quad$ air flow rate complex:

$$
G_{\mathrm{cc}}=\frac{G_{\mathrm{f}} \sqrt{T_{\mathrm{c}}^{*}}}{p_{\mathrm{c}}^{*}}
$$

- $\quad$ air mass flow rate;

- $\quad$ the air flow rate reduced to the parameters behind the compressor:

$$
G_{\mathrm{f} 1 \mathrm{red} \mathrm{c}}=\frac{G_{\mathrm{fI} 0}}{\pi_{\mathrm{c} \Sigma}^{* 5 / 6}}
$$

There is another approach to the determination of concept of small-size known from theory of GTE [3]. The specific weight was considered in [3] as a function of the characteristic size of the engine (D):

$$
\gamma_{\text {engine }}=\frac{M_{\text {engine }}}{N} \approx f\left(\frac{D^{3}}{D^{2}}\right)=f(D),
$$

where $\gamma_{\text {engine }}$ - engine specific weight, $M_{\text {engine }}$ - engine weight, $\mathrm{N}$ - engine power, D - linear engine size.

In paper [4] graphical representation of the dependence (4) is shown, where we can see that theoretical graph coincides with statistical data only in a certain range (fig. 1). This is due to the fact that as the size of the GTE decreases, not all nodes and systems manage to scale proportionally.

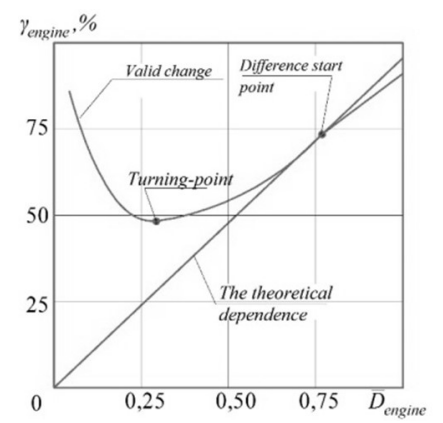

Fig 1. Dependence of specific weight $\left(\gamma_{\text {engine }}\right)$ on the relative diameter of the engine.

Hence, starting with some dimensions of the CC, when they are further reduced, the proportionality in the interrelation between the parameter of the cycle of the gas turbine engine $\left(\pi_{\mathrm{c}}\right)$ and the optimal conditions for the workflow in the $\mathrm{CC}\left(\tau_{\mathrm{ts}}\right)$ is violated.

As the main functional and technological reasons that limit the dimensions of combustion chambers small-sized GTE can act [4,5]:

- technological tolerances for the dimensions of all elements of the $\mathrm{CC}$;

- the sizes of channels for fuel supply;

- dimensions of mixing elements - burners (nozzles)

- gaps between moving elements;

- diameter of fuel droplets;

- the thickness of the gas curtain near the walls of the flame tube; 
- non-uniformity of distribution of parameters and substances along the cross-section of the flame tube.

On the other hand, a number of parameters do not change their size with decreasing dimensions of the $\mathrm{CC}$ :

$$
\tau_{\text {burn }} \geq \tau_{\text {cr }}+\tau_{\text {evap }}+\tau_{\text {mix }}
$$

where $\tau_{\text {burn }}, \tau_{\mathrm{cr}}, \tau_{\mathrm{evap}}, \tau_{\mathrm{mix}}$ - times of burning, chemical reaction, evaporation and mixing, respectively.

The geometric limitations imposed on the choice of the scheme and design of the CC are also dictated by the ratio of the diametrical dimensions of the last stage of the compressor, the first stage of the turbine and the axial dimension between them. These parameters determine the length of the combustion chamber, the angle of its tilt, the overall size of the body and other geometric characteristics. Since one of the basic requirements for the organization of the workflow of the $\mathrm{CC}$ is to create conditions for flame stabilization and ensure high completeness of combustion in the primary zone, it can be argued that the limitations on the dimensions imposed by the compressor and turbine on the geometry of the $\mathrm{CC}$ are ultimately restrictions on the organization of its workflow.

For the sustainable combustion in each design of $\mathrm{CC}$ requires a certain set of structural elements (nozzles, swirlers, etc.) with a certain mutual arrangement and a combination of sizes. At the same time, each of the constructive ways of organizing combustion has its own peculiarities and, accordingly, the possibilities of using it in each specific case for implementing the corresponding scheme of the workflow.

At present, various designs of the flame tube are used to distribute fuel through the primary air layers: with a swirler, a hemispherical front device of the louver type, and several others.

As an alternative to these methods of organizing combustion, a method of forming a toroidal recirculation zone can be used, with a rotating vortex capable of stabilizing the focus of the flame [4]. This method allows us to: use cheaper and easier to manufacture jet nozzles as the main ones, reduce the height of the flame tube and increase the compactness of the compressor.

The authors in [4] provides a theoretical computation of the justification for this method of organizing combustion. It reduces to determining the criterion for stable combustion in a $\mathrm{CC}$ by means of the ratio of the energy required for heating the mixture $E_{\mathrm{r}}$ and the energy available for this process $E_{\mathrm{a}}$.

$$
\begin{aligned}
& E_{\mathrm{e}}=C_{1} \cdot\left(D_{\text {burn }}\right)^{3}, \\
& E_{\mathrm{r}}=C_{2} \cdot\left(D_{\text {burn }}\right)^{2} .
\end{aligned}
$$

From this we obtain the relation

$$
\bar{E}_{r}=\frac{E_{r}}{E_{e}}=\frac{C_{3}}{D_{b u r n}} .
$$

From which it can be seen that minimization of the autonomous combustion source in the CC has a limit in the form $\bar{E}_{\mathrm{n}}=1$.

When comparing the volume of the reverse flow zone of an individual burner and combustion chamber with a toroidal recirculation zone, if we assume that an individual burner (vortex or other type) creates a cylindrical combustion zone with dimensions $D_{\mathrm{bf}}=C_{4} h_{\mathrm{ft}}$ ( $h_{\mathrm{ft}}-$ height flame tube) and $l_{\mathrm{bf}}$. Then 


$$
\left(V_{\mathrm{bf}}\right)_{c i l}=C_{5} \cdot l_{\mathrm{bf}} \cdot \frac{\pi \cdot\left(h_{\mathrm{ft}}\right)^{2}}{4}
$$

Then, if we know the coefficient $\mathrm{C}_{5}$, we will be able to determine the maximum value of the height of the flame tube $h_{\mathrm{ft}}$, to which it is possible to reduce the dimensions of the CC without risk of reaching the limit (7). This value is the boundary in size of the burner $\left(D_{\text {burn }}\right)_{\min }$ and approximately equal to $15-20 \mathrm{~mm}[4,6]$.

To go beyond this limitation, you can combine the discrete combustion zones with the volume $\left(\mathrm{V}_{\mathrm{bz}}\right)_{\mathrm{cyl}}$, located along the ring around the axis of the engine, into a single annular combustion zone (fig. 2). If we assume that the combustion zone of such a "burner" has the form of a torus, then its size can be written as:

$$
\left(V_{\mathrm{bz}}\right)_{\text {thor }}=C_{6} \cdot 2 \pi R \cdot \frac{\pi \cdot\left(h_{\mathrm{ft}}\right)^{2}}{4}
$$

where $R$ - large radius of the torus, and $h_{\mathrm{ft}}$ is the height of the flame tube.

However, the limits of flame stabilization can be considered in other ways. Thermal theory:

$$
\frac{q_{2}}{q_{1}}=\frac{w^{\mathrm{n}} d^{\mathrm{n}}\left(T_{\mathrm{mg}}-T_{0}\right)}{v^{\mathrm{n}} w\left(T_{\mathrm{f}}-T_{0}\right)}=\text { const },
$$

where $q 1$ - amount of heat transmitted by the fresh mixture from the combustion products of the circulation zone, $q 2$ - amount of heat required by the fresh mixture for ignition. Contact theory

$$
\frac{t_{1}}{t_{2}} \sim \frac{d \cdot U_{\mathrm{H}}^{2}}{w \cdot a_{\mathrm{m}}}=\mathrm{Mi}
$$

This theory is based on the ratio of the times of contact of the fresh mixture with the hot combustion products in the recirculation zone $t_{1}$ and the time necessary for heating the mixture to the temperature required for ignition. Reactor theory is also existed [5].

On the basis of these theories, we can assume that stabilization mainly depends on the speed of air and fuel-air mixture, the thermal diffusivity, the ignition temperature and the temperature of the mixture, and also on the size of the recirculation mixing zone. If all other parameters are assumed to be constant, a zone with a larger size is obtained with greater stability, which makes it possible to provide either a reserve of available energy or to form an area in which there will be a longer contact time.

If we consider the flow in a scheme with a swirler and a toroidal one, then if the holes of the first row are identical, it is evident that in the second case preconditions exist for the organization of a more developed combustion zone (fig. 2).
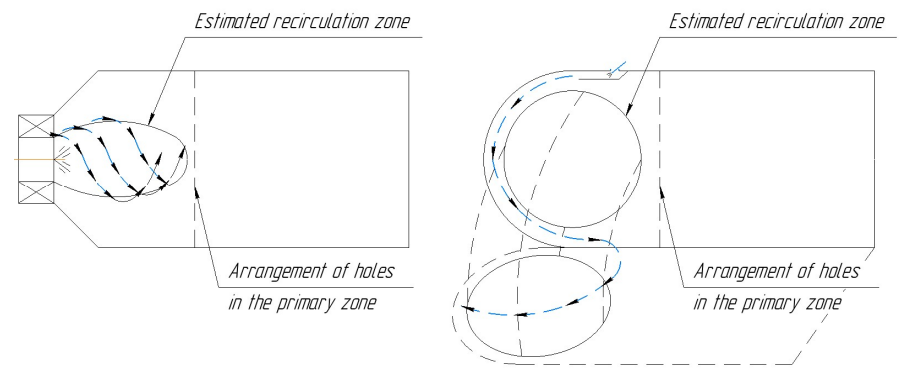

Fig. 2. Comparison of particle tracks of different recirculation zones.

Thus, the toroidal form of the combustion zone has the advantage from the point of ensuring stable combustion. However, its application raises a number of new tasks related to the dynamics of gas flow, the search for optimal ways of preparing fuel assemblies and with 
questions of energy-mass transfer. Therefore, for the further investigation, numerical modeling of the working process was used.

On the basis of the above theoretical calculations, within the implementation of one of the projects, a combustion chamber was developed, the initial shape of which is shown in fig. 3.

Further, the investigation of the $\mathrm{CC}$ workflow will be conducted by simulation method to prove the toroidal scheme of combustion.

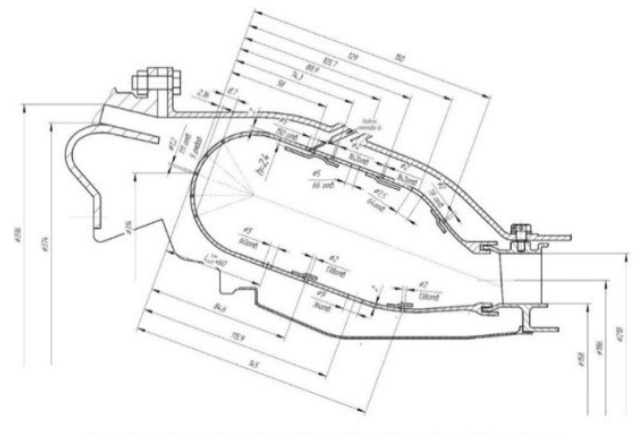

Fig. 3. The general view of CC.

\section{Discussion}

The toroidal recirculation zone, with the correct organization of combustion, can contribute to a more uniform exit of hot gas from the back-current zone, due to the absence of a discrete flame source, and accordingly to a more uniform temperature field in the circumferential direction at the outlet from the CC.

The comparison of the dependences of the volume of combustion zones for two types of combustion organization showed that the toroidal recirculation zone makes it possible to reduce the diametrical dimensions of the flame tube with the same $\mathrm{V}_{\mathrm{bz}}$. This flame tube design allows forming the toroidal recirculation-mixing that leads to increasing the path of burning mixture.

In addition, the lack of the traditional flame tube head will reduce the cost of production of small-sized GTD.

This work was supported by the Ministry of education and science of the Russian Federation in the framework of the implementation of the Program "Research and development on priority directions of scientific-technological complex of Russia for 2014-2020" (RFMEFI58716X0033).

\section{References}

1. V.A. Grigorev, V.V. Zrelov, Yu.M. Ignatkin, Vertoletnie gazoturbinnie dvigateli, 490 (2007).

2. V.S. Kuz'michev, Vestnik Samarskogo universiteta. Aehrokosmicheskaya tekhnika, tekhnologii i mashinostroenie, 2, 15 (2016).

3. A. Dzhadzh, Gazoturbinnye dvigateli maloj moshchnosti, 270 (1963).

4. V.P. Lukachyov, Vestnik Samarskogo universiteta. Aehrokosmicheskaya tekhnika, tekhnologii i mashinostroenie, 1, 1-15 (1998).

5. A.M. Lanskij, Metody i sredstva povysheniya ehffektivnosti rabochego processa kamer sgoraniya malorazmernyh GTD: diss. (2014)

6. A.M. Lanskij, S.V. Lukachev, S.G. Matveev, SNC RAN, 2, 1-11 (2009) 